P. Holický, Charles University, Faculty of Mathematics and Physics, Department of Mathematical Analysis, Sokolovská 83, 18675 Prague 8, Czech Republic. email: holicky@karlin.mff.cuni.cz

C. E. Weil, Mathematics Department, Michigan State University, East Lansing, MI 48824-1027, USA. email: weil@math.msu.edu

L. Zajíček, Charles University, Faculty of Mathematics and Physics, Department of Mathematical Analysis, Sokolovská 83, 18675 Prague 8, Czech Republic. email: zajicek@karlin.mff.cuni.cz

\title{
A NOTE ON THE DARBOUX PROPERTY OF FRÉCHET DERIVATIVES*
}

\author{
Dedicated to the memory of Casper Goffman
}

\begin{abstract}
Let $A$ be a subset of a Banach space $X$ and $f$ a Fréchet differentiable function on $A$ (with respect to $A$ ). We give a simple proof of the connectedness of the graph of $f^{\prime}$ in $X \times X^{*}$ under relatively weak conditions on $A$. In particular, we simplify a proof by J. Malý of the connectedness of the range of $f^{\prime}$ for some convex sets $A$. At the same time, we extend an older result of C. E. Weil on the connectedness of the range of $f^{\prime}$ for some non-convex sets $A \subset \mathbb{R}^{n}$.
\end{abstract}

\section{Introduction.}

Throughout the entire article, $X$ will always be a real Banach space. J. Malý [Ma, Theorem 1.4] proved that the Fréchet derivative of a differentiable function on a convex subset of $X$ with nonempty interior has connected range in its dual space $X^{*}$ endowed with its dual norm. Refining the proof of J. Malý,

Key Words: Darboux property, porosity, Fréchet derivative

Mathematical Reviews subject classification: 26B06

Received by the editors January 2, 2007

Communicated by: B. S. Thomson

* The work was partly supported by the research project MSM 0021620839 financed by MSMT, by GAČR 201/06/0198, and by GAČR 201/06/0018. 
C. Weil observed that the same conclusion holds also for some non-convex subsets of $\mathbb{R}^{n}$ (unpublished).

More recently, T. Mátrai used the result of J. Malý to get a Darboux property of the Gâteaux derivative of locally Lipschitz functions on separable Banach spaces, (see [Má]). His procedure uses explicitly that the Gâteaux derivative of a Lipschitz function is of the first Baire class as a function from a separable Banach space $X$ to its dual $X^{*}$ endowed with the weak-star topology $w^{*}$. His conclusion is that not only the range of the Gâteaux derivative but even its graph is a connected subset of $X \times\left(X^{*}, w^{*}\right)$.

The aim of our note is twofold.

a) Using some of Mátrai's ideas and an easy lemma on porosity (Lemma 2), we simplify the proof of J. Malý and we get the connectedness even of the graph of the Fréchet derivative as a subset of $X \times X^{*}$ endowed with the product topology corresponding to the norm on $X$ and to its dual norm on $X^{*}$.

b) We extend the older unpublished results of Weil on the range of the Fréchet derivative on non-convex sets to infinite-dimensional Banach spaces in Theorem 1, which provides a generalization of Malý's result.

To formulate our main result, we recall the notions of a relative derivative and of porosity.

Definition 1. Let $B \subset X$ be non-empty without isolated points. Let $f: B \rightarrow$ $\mathbb{R}$ be given. We say that $g: B \rightarrow X^{*}$ is a (relative) Fréchet derivative of $f$ on $B$ if, for each $a \in B$,

$$
\lim _{\substack{x \rightarrow a \\ x \in B}} \frac{|f(x)-f(a)-g(a)(x-a)|}{\|x-a\|}=0 .
$$

Of course, $g$ is not always uniquely determined, but we will use the notion of the relative derivative only under assumptions, which imply the uniqueness (see Remark 1).

Definition 2. A set $E \subset X$ is (upper) porous at $a \in X$ if there are $c>0$ and $x_{n} \in X, x_{n} \neq a$ with $x_{n} \rightarrow a$ such that $B\left(x_{n}, c\left\|x_{n}-a\right\|\right) \cap E=\emptyset$, where $B(x, r)$ denotes the open ball in $X$ with radius $r>0$ which is centered at $x \in X$.

Further, the dual space $X^{*}$ of $X$ is endowed with the corresponding dual norm.

Our main result reads as follows.

Theorem 1. Let $\emptyset \neq B$ be a subset of a Banach space $X$ such that int $B$ is connected, and $X \backslash \operatorname{int} B$ is porous at every $x \in B \cap \partial B$. Let $g: B \rightarrow X^{*}$ be a (relative) Fréchet derivative of a function $f: B \rightarrow \mathbb{R}$ on $B$. Then the graph of $g$ is a connected subset of $X \times X^{*}$. In particular, $g(B)$ is connected in $X^{*}$. 
This theorem is an almost immediate consequence of Proposition 1 and Lemma 1 below. (See the proof at the end of this paper.) As an immediate corollary we obtain the (slightly generalized) result due to Malý.

Corollary 1. Let $B \subset X$ be a convex set with int $B \neq \emptyset$. Let $g: B \rightarrow X^{*}$ be the (relative) Fréchet derivative of a function $f: B \rightarrow \mathbb{R}$ on $B$. Then the graph of $g$ is a connected subset of $X \times X^{*}$. In particular, $g(B)$ is connected in $X^{*}$.

Proof. Let $x \in \partial B$ be given. Choose a ball $B(z, r) \subset \operatorname{int} B$. Denote by $C$ the convex hull of $\{x\} \cup B(z, r)$, and observe that $X \backslash C$ is clearly porous at $x$.

Remark. 1. It is not difficult to observe that, under the assumptions of Theorem $1, g$ is uniquely determined.

2. It is not sufficient to assume in Theorem 1 that B is connected (instead of the stronger assumption that int $B$ is connected). If $G \subset \mathbb{R}^{2}$ is defined by $G=G_{1} \cup G_{2}$, where $G_{1}=\left\{(x, y) \in \mathbb{R}^{2}: x<0\right\}$ and $G_{2}=\left\{(x, y) \in \mathbb{R}^{2}: x>\right.$ 0 and $\left.y \in\left(-x^{2}, x^{2}\right)\right\}, f(x, y)=0$ for $(x, y) \in G_{1} \cup\{(0,0)\}$, and $f(x, y)=y$ on $G_{2}$, then the Fréchet derivative $g$ of $f$ on $B=G \cup\{(0,0)\} \subset \operatorname{cl} G=\operatorname{cl}$ int $B$ exists, but $g(B)=\{(0,0),(0,1)\} \subset\left(\mathbb{R}^{2}\right)^{*}$ is not connected. Moreover, it can be shown that $f$ can be extended to a differentiable function $\tilde{f}$ on $\mathbb{R}^{2}$. Then $(\tilde{f})^{\prime}(B)=g(B)$ is not connected.

\section{A Lemma on Derivatives at Boundary Points.}

The proof of the following lemma, which is an easy consequence of the Ekeland principle, is essentially contained in the proof of [HMWZ, Theorem 5].

Lemma 1. Let $X$ be a Banach space, $G \subset X$ open, $a \in \partial G$, and let $X \backslash G$ be porous at a. Let $M:=G \cup\{a\}$ and suppose that $g: M \rightarrow X^{*}$ is a (relative) Fréchet derivative of a function $f: M \rightarrow \mathbb{R}$ on $M$. Then $(a, g(a))$ belongs to the closure of the graph of $\left.g\right|_{G}$ in $X \times X^{*}$. In particular, $g(a) \in \operatorname{cl} g(G)$.

Proof. There exists a $c>0$ and a sequence $x_{n} \in G$ tending to $a$ such that the open balls $B\left(x_{n}, c\left\|x_{n}-a\right\|\right)$ are subsets of $G$. We may and do suppose that $g(a)=0 \in X^{*}$. Let us fix an arbitrary $\omega>0$. It is sufficient to find $z \in G$ such that

$$
\|z-a\|<\omega \text { and }\|g(z)\|<\omega .
$$

To this end choose $\eta>0$ so small that $4 \eta(1+c) \omega^{-1}<c$. Since $g(a)=0$ and $\left\|x_{n}-a\right\|$ tends to zero, we can choose $n \in \mathbb{N}$ so large that, for each 


$$
\begin{aligned}
& z \in B\left(x_{n}, c\left\|x_{n}-a\right\|\right), \\
& \quad|f(z)|<\eta\|z-a\| \leq \eta\left(\left\|z-x_{n}\right\|+\left\|x_{n}-a\right\|\right) \leq \eta(1+c)\left\|x_{n}-a\right\| .
\end{aligned}
$$

Using the Ekeland principle (namely, we may apply [Ph, Lemma 3.13] with $E:=X, f$ defined/redefined by $f(x)=\infty$ for $x \in X \backslash \operatorname{cl} B\left(x_{n}, c\left\|x_{n}-a\right\|\right)$, $\varepsilon:=2 \eta(1+c)\left\|x_{n}-a\right\|, \lambda:=\omega / 2$, and $x_{0}:=x_{n}$ due to (3)), we obtain a point $z \in \operatorname{cl} B\left(x_{n}, c\left\|x_{n}-a\right\|\right)$ such that

$$
\frac{f(x)-f(z)}{\|x-z\|}>-\frac{\omega}{2} \text { for each } x \in \operatorname{cl} B\left(x_{n}, c\left\|x_{n}-a\right\|\right) \backslash\{z\},
$$

and

$$
\left\|z-x_{n}\right\| \leq \frac{2 \eta(1+c)\left\|x_{n}-a\right\|}{\omega / 2}<c\left\|x_{n}-a\right\| .
$$

Consequently, $z \in B\left(x_{n}, c\left\|x_{n}-a\right\|\right)$, and so $\|z-a\|<\omega$ by (2), and (4) easily implies that $\|g(z)\|=\left\|f^{\prime}(z)\right\|<\omega$. Thus, (1) holds.

\section{Darboux Property on Open Sets.}

We will need the following lemma which is an easy modification of the wellknown Proposition 2.24 of [Za].

Lemma 2. Let $(Y, \rho)$ be a metric space such that every open ball $B(z, r)$, with $z \in Y$ and $r>0$, is connected and let $H \subset Y$ be open. Then the set $P=\{x \in \partial H: Y \backslash H$ is porous at $x\}$ is a residual set in $\partial H$.

Proof. For each $n \in \mathbb{N}$, let $P_{n}$ be the set of all $x \in \partial H$, for which there exists a ball $B(z, r) \subset H$ such that $r<1 / n$ and $x \in B(z, 2 r)$. It is easy to see that each $P_{n}$ is open in $\partial H$ and $\bigcap\left\{P_{n}: n \in \mathbb{N}\right\} \subset P$. So, it suffices to prove that each $P_{n}$ is dense in $\partial H$. To this end, consider an arbitrary $y \in \partial H$ and $0<\varepsilon<1 / n$. Choose $z \in H \cap B(y, \varepsilon)$ and put $r:=(3 / 4)$ dist $(z, \partial H)$. Since $B(z, r) \cap \partial H=\emptyset$, $z \in H$, and $B(z, r)$ is connected, we get $B(z, r) \subset H$. It is obvious that there is an $x \in B(z, 2 r) \cap \partial H$. Since $\operatorname{dist}(z, \partial H) \leq\|z-y\|<\varepsilon<1 / n$, we have $x \in P_{n}$. Moreover, $\|x-y\| \leq\|x-z\|+\|z-y\|<2 r+\|z-y\| \leq 3\|z-y\|<3 \varepsilon$. As $\varepsilon \in(0,1 / n)$ was arbitrary, this shows that $P_{n}$ is dense in $\partial H$.

We also need the following lemma which generalizes the classical result on derivatives of real functions of a real variable and which can be essentially found in [MS]. Let us point out that an analogous result [Má, Lemma 2.4] was crucial for the main result of [Má]. We recall that by mappings of the first Baire class we mean pointwise limits of sequences of continuous mappings, 
and by mappings of the first Borel class, the mappings whose inverse images of open sets are $F_{\sigma}$.

Lemma 3. Let $G$ be an open subset of a Banach space $X$. Let $f: G \rightarrow \mathbb{R}$ have Fréchet derivative $f^{\prime}(x) \in X^{*}$ at every point of $G$. Then the mapping $\varphi: x \mapsto\left(x, f^{\prime}(x)\right)$ is of the first Borel class from $G$ to $X \times X^{*}$.

Proof. The Fréchet derivative $f^{\prime}: G \rightarrow X^{*}$ is Baire one by [MS, Theorem 1]. It follows in a straightforward fashion that the mapping $x \mapsto\left(x, f^{\prime}(x)\right)$ of $G$ to $X \times X^{*}$ is Baire one as well and so it is of the first Borel class (see, e.g., $[\mathrm{K}, \S 31, \mathrm{VIII}])$.

We now present our simplified proof of (a slightly improved version of) Malý's result for open sets.

Proposition 1. Let $X$ be a Banach space, let $\Omega \subset X$ be a connected open set, and let $f: \Omega \rightarrow \mathbb{R}$ be Fréchet differentiable. Then the graph graph $g$ of the Fréchet derivative $g$ of $f$ is a connected subset of $X \times X^{*}$. In particular, $g(\Omega)$ is connected.

Proof. Let us suppose, to the contrary, that graph $g$ is not connected. Then there are nonempty disjoint sets $\widetilde{A}, \widetilde{B}$ which are clopen in graph $g$ and $\widetilde{\widetilde{A}} \cup \widetilde{B}=$ graph $g$. It easily follows from Lemma 3 that their projections to $X, A:=$ $\pi_{X}(\widetilde{A})=\varphi^{-1}(\widetilde{A})$ and $B:=\pi_{X}(\widetilde{B})=\varphi^{-1}(\widetilde{B})$ are $F_{\sigma}$. Clearly $A \neq \emptyset, B \neq \emptyset$, $\Omega=A \cup B$, and $A \cap B=\emptyset$. Since $\Omega$ is connected, the set $F:=\partial_{\Omega} A=\partial_{\Omega} B$ is nonempty. Using the Baire category theorem in $F$ (which is topologically complete as a $G_{\delta}$ subset of $X$, cf. $\left.[\mathrm{K}, \S 33, \mathrm{VI}]\right)$ we obtain that one of its $F_{\sigma}$ subsets $F \cap A, F \cap B$ (say $F \cap A$ ) has nonempty interior in $F$. Consider a convex open set $\Omega^{*} \subset \Omega$ such that $\emptyset \neq F^{*}:=\Omega^{*} \cap F \subset A$ and set $B^{*}:=B \cap \Omega^{*}$. Since clearly $B^{*} \cap \partial B^{*}=B^{*} \cap \partial_{\Omega} B=B \cap \Omega^{*} \cap F \subset B \cap A=\emptyset$, the set $B^{*}$ is open. Lemma 2 (we may apply it to $Y:=\Omega^{*}$ and $H:=B^{*}$ since every ball in $\Omega^{*}$ is connected due to its convexity), and the Baire category theorem used in $F^{*}=\partial_{\Omega^{*}} B^{*}$, give the existence of $a \in F^{*} \subset A$ such that $\Omega^{*} \backslash B^{*}$ is porous at $a$ in $\Omega^{*}$. Since $\Omega^{*}$ is open, $X \backslash B^{*}$ is porous at $a$ in $X$ as well. Lemma 1 (with $\left.G:=B^{*}\right)$ implies $\left.(a, g(a)) \in \operatorname{clgraph} g\right|_{B^{*}}$. So $(a, g(a)) \in \widetilde{B}$, since $\widetilde{B}$ is closed in graph $g$ and graph $\left.g\right|_{B^{*}} \subset \widetilde{B}$. We obtain a contradiction, since $a \in A$, and so $(a, g(a)) \in \widetilde{A}$.

Proof of Theorem 1. Let $G:=\operatorname{int} B$. We denote the graph of $g$ by $\Gamma$ and the graph of $\left.g\right|_{G}$ by $\Gamma_{0}$. Proposition 1 implies that $\Gamma_{0}$ is connected and Lemma 1 easily implies $\Gamma_{0} \subset \Gamma \subset \operatorname{cl} \Gamma_{0}$. Therefore, $\Gamma$ is connected as well. 


\section{References}

[HMWZ] P. Holický, J. Malý, C. Weil and L. Zajíček, A Note on the Gradient Problem, Real Analysis Exchange, 22 (1996/97), 225-235.

[K] K. Kuratowski, Topology, Vol I, Academic Press, New York 1966.

[Ma] J. Malý, Darboux Property for Gradients, Real Analysis Exchange, $22(1996 / 97), 167-173$.

[MS] E. Matoušková and Ch. Stegall, The Structure of the Fréchet Derivative in Banach Spaces, Vienna, Preprint ESI 1014 (2001), http://www.esi.ac.at.

[Má] T. Mátrai, Graphs of Gâteaux Derivatives are $w^{*}$-connected, Real Analysis Exchange, 29 (2003/04), 291-297.

[Ph] R. R. Phelps, Convex Functions, Monotone Operators and Differentiability, Lecture Notes in Math., 1364, Springer-Verlag, Berlin, 1993.

[Za] L. Zajíček, Porosity and $\sigma$-porosity, Real Analysis Exchange, 13 (1987/88), 314-350. 